\title{
Opportunistic Relay Selection for Rateless-Coded Cooperative Relaying
}

\author{
Jietao Zhang, Hongcheng Zhuang, Zezhou Luo \\ Communication Technologies Research Department \\ Huawei Technologies Co., Ltd. \\ Shenzhen, China 518129 \\ \{jtzhang, zhc, luozezhou\}@huawei.com
}

\author{
Yun Li \\ Special Research Centre for Optical Internet and Wireless \\ Chongqing University of Post and Telecommunications \\ Chongqing, China \\ liyun@cqupt.edu.cn
}

\begin{abstract}
Cooperative relaying has the potential to enhance wireless link throughput, while rateless-coded transmission adapts to the wireless channel automatically. The combination of both holds promising performance enhancement for practical wireless communications. Relay selection is a challenging issue in conventional cooperative communications and attracts extensive research. However, there is little literature on the investigation of relay selection in the context of rateless-coded cooperative relaying. We argue that the relay selection scheme for the conventional cooperative relaying is suboptimal when being applied to the rateless-coded cooperative relaying due to their fundamental differences in signal processing. In this paper, we propose a relay selection scheme specifically for rateless-coded cooperative relaying. The relay selection protocol is devised and the associated selection criterion is derived. Simulation results demonstrate that the proposed relay selection scheme outperforms other existing ones in literature, therefore justifying our arguments.
\end{abstract}

Keywords- cooperative relaying; rateless-coded transmission; relay selection

\section{INTRODUCTION}

Cooperative relaying, in which one or more relays assist a source in forwarding its message to a destination, emerges as a promising technique to combat fading in wireless network, so as to achieve diversity gain as well as reducing power consumption. There have been extensive ongoing research activities to develop cooperative relaying protocols and resource management techniques to exploit its potential benefits [1]-[3]. In the conventional cooperative relaying, fixed-rate code is employed for transmission, in which energy of orthogonal transmissions from different nodes is combined by the receiver. The problem with such fixed-rate coding is that the outage probability of transmission is never zero without precise channel state information (CSI) at the transmitter. In comparison to fixed-rate codes, rateless codes [4], as what it sounds, do not have a fixed rate for transmission. Instead, the transmission adapts the rate "on the fly" to the channel status, and requires no knowledge of CSI or even channel statistics at the transmitter [5]. According to the principle of rateless codes, the transmitter sends rateless-coded packets, and the receiver keeps accumulating mutual information from the transmitter until it becomes capable of successfully decoding the transmitted message [6]. In the event that the destination successfully decodes, it sends an acknowledgement to inform the transmitter so that the transmitter can terminate ratelesscoded transmission of the data packet and starts next data packet transmission.

Merits of rateless codes in relaying schemes have been demonstrated by many research works [5]-[11]. Among these works, a simplified model consisting of three nodes, i.e. a source, a relay and a destination, is investigated [5][7][8]. This configuration forms the building block for all relay networks but should be generalized into practical networks. In [6], several multi-relay quasi-synchronous and asynchronous rateless coded schemes are proposed. In a practical network setting with multiple relays, single relay selection is an important research issue that has attracted many research efforts in the conventional cooperative communications [12][14] [14], due to its simplicity and yet considerably large energy efficiency and full diversity gain. Relay selection has also been discussed in the context of rateless-coded cooperation in recent literature [8]-[11]. A power scheduling scheme is proposed in [8] to turn on or off the power of relay, in an attempt to opportunistically utilize relay for data forwarding. A selection-based rateless coded cooperative protocol is proposed in [9], in which among a set of relays that can decode the source's information one relay having a best relay-destination link is effectively selected as the desired relay to participate in cooperation. A relay selection scheme similar to [12] is adopted in [10][11] for rateless-coded cooperative relaying. In these schemes, the relay selection criterion is essentially identical to that used for the conventional fixedrated cooperative relaying. We argue that due to the difference in information collection manners between the rateless-coded cooperation and the conventional cooperation, the relay selection criterion should also be fundamentally different. The relay selection scheme used for conventional cooperation through energy accumulation is suboptimal when being applied to rateless-coded cooperation through mutual information accumulation. Therefore, the optimal selection scheme specifically appropriate for rateless-coded cooperative relaying is desired in order to fully explore its potentials.

The contribution of this paper is that we propose a relay selection scheme specifically for rateless-coded cooperative relaying. The scheme consists of two parts. In the first part, we devise a relay selection protocol, in which a relay that could optimally help the destination accumulate mutual information is selected to participate in cooperation. To facilitate the 
selection scheme, a relay selection criterion is then derived in the second part. The criterion is fundamentally different from that used in the conventional cooperation scheme. Compared with existing relay selection schemes in the literature, either applied to conventional or rateless-coded cooperation, our proposed scheme outperforms them by giving rise to higher channel capacity.

The remainder of the paper is organized as follows. In Section II, we describe the rateless-coded cooperation system model in this study, and present the proposed relay selection protocol. The associated relay selection criterion for the protocol is then derived and described in Section III. In Section IV, we present some simulation results of our proposed scheme in comparison with other existing schemes. This is followed by the conclusion in Section V.

\section{RELAY SELECTION PROTOCOL}

The system model of consideration here is a sourcedestination pair with multiple relay candidates in between, as illustrated in Figure 1. which is common practice in the literature. In this section, we devise the relay selection protocol for rateless-coded cooperative relaying, which is substantially different from that for the conventional cooperative relaying [12]-[14][14]. In the conventional cooperative relaying, essentially the relay nodes or (and) the destination performs channel measurements on the corresponding wireless links, and feedback(s) to the source to conduct relay selection in a centralized way [13], or in a semi-distributed way with less feedback overhead [14], or in a fully distributed way without feedbacks [12]. Since mutual information accumulation rather than energy accumulation is performed by receiving nodes in rateless-coded cooperative relaying, the receiver processing mechanism as well as the relationship of link capacity with respect to channel quality for the conventional cooperative relaying does not hold anymore. As a result, a new relay selection protocol as well as selection criterion for conventional cooperative relaying are required to cope with the rateless-coded cooperative relaying. We devise an appropriate relay selection protocol, which is schematically sketched in Figure 1. Five steps are taken for the destination to obtain knowledge of the source and relay nodes, and to determine and select the best relay, which are described as follows.

1) After synchronization of all nodes, at Step 1, the source (S) transmits a request message using rateless codes $(\mathrm{ml}$ message depicted in Figure 1. ), the destination (D) and surrounding relay nodes $\left(\mathrm{R}_{\mathrm{i}}\right.$, with $i$ denoting the index of relay node) keep accumulating respective received information and attempt to decode the request message.

2) At step 2, any node that successfully decodes the message then broadcasts a feedback message ( $A C K{ }_{1} R x$ message depicted in Figure 1. ) to inform the source of reception. Before its own successful decoding, the destination can also receive individual feedbacks from relay nodes that can decode before itself. The destination stores the reception times of individual feedbacks from relay nodes. At the point of successful decoding, the destination also sends back a feedback message ( $A C K 1 \_D$ message depicted in Figure 1. ) to the source, so that the source terminates transmitting the rateless-coded request message.

3) At Step 3, after feeding back to the source, the destination initiates a response message $(m 2$ message depicted in Figure 1. ). The source and surrounding relay nodes keep accumulating respective received information and attempt to decode the response message.

4) As a counterpart of Step 2, at Step 4, any relay that can decode before the transmitter sends a feedback message ( $A C K 2_{2} R x$ message depicted in Figure 1. ) to inform the destination of reception. The destination stores the reception times of individual feedbacks from relay nodes before that from the source $(A C K 2 \leq S$ message depicted in Figure 1. ) and then terminates transmitting the ratelesscoded response message.

5) At Step 5, by our proposed relay selection criterion, the destination then determines a best relay from the available relay nodes according to the reception times of feedback messages from Step 2 and Step 4. Afterwards, the destination sends the acknowledgement message to both the source and the selected relay $\left(A C K 3 \_D\right.$ message depicted in Figure 1. ) for cooperation in subsequent data transmissions.

To further illustrate the selection procedure, we note that in Figure 1. , R4 cannot decode the $m l$ message before the destination does due to its channel to the source has lower quality than that between the source and the destination. Therefore, when the destination sends the $A C K 1 \_D$ message, the source terminates transmitting the rateless-coded $m l$ message. As a result, R4 can no longer accumulate information for decoding the $m 1$ message, thus it loses the chance to become a potential relay and remains silent during the subsequent selection procedure and cooperation round. Likewise, in Figure 1., R1 cannot decode the $m 2$ message before the source does and thus becomes unavailable either. During the abovementioned procedure, to avoid potential collisions of feedback messages from different nodes, a contention minislot concept similar to [15] could be utilized to alleviate the situation. The relay selection protocol devised above can ensure that any relay selected from the relay candidates experiences better source-relay and relaydestination channel qualities than the source-destination channel, which ensures that the relay so selected can definitely yield positive cooperation gain during the data transmission phase.

\section{RELAY SELECTION CRITERION}

With the knowledge of reception times of individual feedbacks from both the source and the positive relay nodes, the destination determines the decoding times of $m 1$ and $m 2$ of the source and positive relay nodes. To handle the relay selection, we propose a selection criterion. Since mutual information accumulation rather than energy accumulation is performed by receiving nodes in rateless-coded cooperative relaying, the relay selection criterion for conventional cooperative relaying needs to be modified accordingly. 


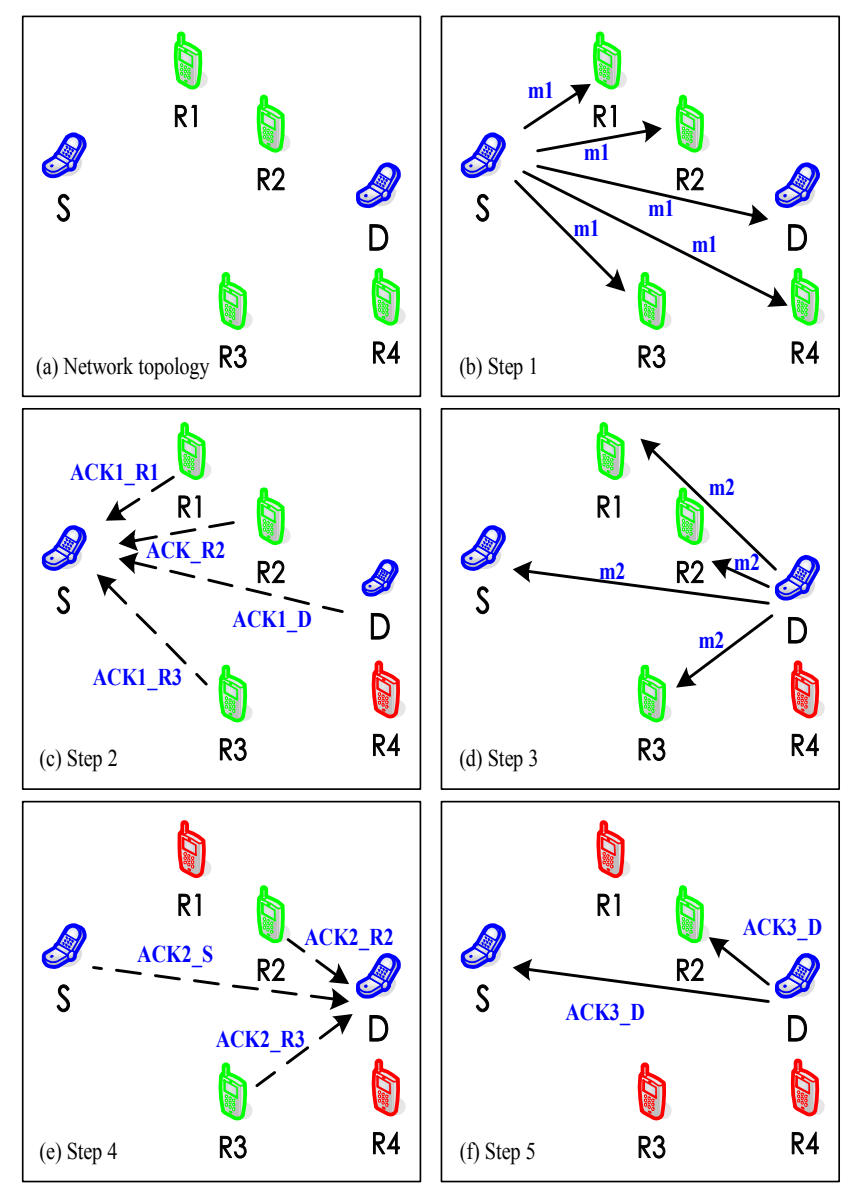

Figure 1. Network topology and relay selection procedure.

For a two-hop cooperative relaying, we assume the channel SNR of the S-D link, that of the S-R link and that of the R-D link are denoted as $\gamma_{0}, \gamma_{1}$ and $\gamma_{2}$, respectively. Thus, given data bits of $a$ for transmission, the channel uses, i.e. times for correctly decoding data, for the three links can be calculated as $t_{0}=a / c_{0}, t_{1}=a / c_{1}$, and $t_{2}=a / c_{2}$, respectively, where $c_{0}=\log _{2}\left(1+\gamma_{0}\right), c_{1}=\log _{2}\left(1+\gamma_{1}\right)$, and $c_{2}=\log _{2}\left(1+\gamma_{2}\right)$.

In our system model, assume that one of the relay nodes is selected for cooperative relaying and is denoted as $R_{i}$, and the channel uses taken by $\mathrm{R}_{\mathrm{i}}$ to decode the transmitted data from $\mathrm{S}$ is denoted as $t_{i 1}$. During the data reception of $\mathrm{R}_{\mathrm{i}}, \mathrm{D}$ also receives and attempts to decode the transmitted data. The accumulated mutual information of $\mathrm{D}$ during the first phase (i.e., $\mathrm{R}_{\mathrm{i}}$ reception phase) can be calculated as $I_{\text {phase } 1}=t_{\text {phase } 1}^{i} \cdot c_{0}$, with reception time $t_{\text {phase }}^{i}=t_{i 1}$, and the remaining information to be accumulated during the second phase (i.e., $\mathrm{R}_{\mathrm{i}}$ transmission phase) is $I_{\text {phase } 2}=a-I_{\text {phase } 1}$.

After $\mathrm{R}_{\mathrm{i}}$ 's successful decoding of the transmitted data, it reencodes the data and transmits to $\mathrm{D}$ in the second phase, thus the channel uses by $\mathrm{D}$ for decoding the transmitted data from $R_{i}$ is given by

$$
\begin{aligned}
t_{\text {phase } 2}^{i}=\frac{I_{\text {phase } 2}}{c_{2}} & =\frac{a-t_{i 1} c_{0}}{c_{2}} \\
& =\frac{\left(t_{0}-t_{i 1}\right) c_{0}}{c_{2}} \\
& =\frac{\left(t_{0}-t_{i 1}\right) t_{i 2}}{t_{0}}
\end{aligned}
$$

The total channel uses by $\mathrm{D}$ to successfully decode the data transmitted from $\mathrm{S}$ with assistance of $\mathrm{R}_{\mathrm{i}}$ is thus given by

$$
t_{d}^{i}=t_{\text {phase } 1}^{i}+t_{\text {phase } 2}^{i}=t_{i 1}+\frac{\left(t_{0}-t_{i 1}\right) t_{i 2}}{t_{0}}
$$

Therefore, to achieve the highest transmission rates between $\mathrm{S}$ and $\mathrm{D}$ is equivalent to selecting an $\mathrm{R}_{\mathrm{i}}$ which gives rise the minimum value of $t_{d}^{i}$. As a result, the relay selection criterion of our scheme is derived to be

$$
R_{i}^{*}=\arg \min _{R_{i} \in R C}\left\{t_{i 1}+t_{i 2}\left(1-\frac{t_{i 1}}{t_{0}}\right)\right\}
$$

where $R_{i}^{*}$ denotes the optimal relay selected for cooperation and $R C$ denotes the set of relay nodes. By some manipulative operations, it can be easily proved that $t_{d}^{i}$ is always smaller than $t_{0}$, which indicates that $R_{i}^{*}$ can always offer better performance than direct transmission. It is noted that the proposed criterion fundamentally differs from the criterion used in the conventional relaying [11][12]. This is due to the fact that in the rateless-coded cooperation, mutual information rather than energy is accumulated at the destination.

\section{SimUlation Results}

The simulation scenario in our study consists of a pair of source and destination with sixteen relay candidates located in between. The distance between the source and the destination is normalized to unity, and the relay nodes are randomly distributed around the midpoint of the line connecting the source and the destination. Channels between all nodes are assumed to be frequency-flat block-fading Rayleigh channels. The noise at the receiver is assumed to be complex Gaussian distributed with the mean zero and variance one.

Figure 2 and Figure 3 show the capacity comparison between rateless-coded and conventional fixed-rated cooperative transmissions, at low and high SNR regimes, respectively. In both figures, Rateless_Coop refers to the achievable capacity of rateless-coded cooperative transmission by our proposed relay selection scheme; Conv_Coop refers to that of conventional cooperative transmission by the conventional relay selection scheme [11][12]; Direct refers to the direct transmission scheme. In Figure 2 we observe the following three features. Firstly, at low SNR regime both rateless-coded and conventional cooperative transmission result in higher capacity compared to the direct transmission. This is expected since cooperative relaying, either mutual information or energy accumulation, benefits from both power 
and diversity gains. Secondly, the rateless-coded cooperation constantly outperforms the conventional cooperation. This is mainly due to the fact that by virtue of rateless-coded transmission, mutual information, i.e. information bits, can be accumulated directly for decoding, which is more spectrumefficient than the energy accumulation of the conventional cooperation. Finally, with increasing SNR, performance gain of the conventional cooperation over the direct transmission is reduced, which is due to the multiplexing loss subject to the half-duplex relay constraint. In contrast, rateless-coded cooperation does not have such performance loss.

In Figure 3 we observe the following two features. Firstly, since the multiplexing relay loss associated with the conventional cooperative transmission has been taken into account in the relay selection, no relay will be selected at high SNR regime, hence the conventional cooperative transmission in effect reverts to the direct transmission. Secondly, in contrast to the conventional cooperation, the rateless-coded cooperation constantly outperforms the direct transmission. This indicates that even at high SNR regime, an appropriate relay can be selected by our proposed scheme for beneficial cooperation.

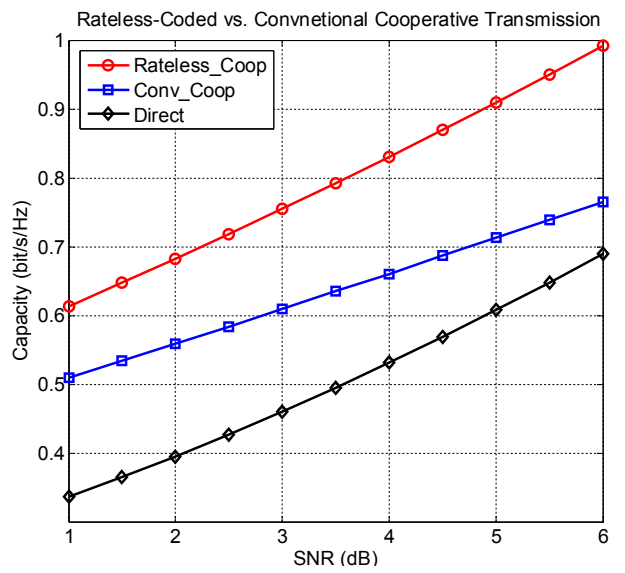

Figure 2. Capacity comparison between rateless-coded and conventional cooperative transmission at low SNR regime.

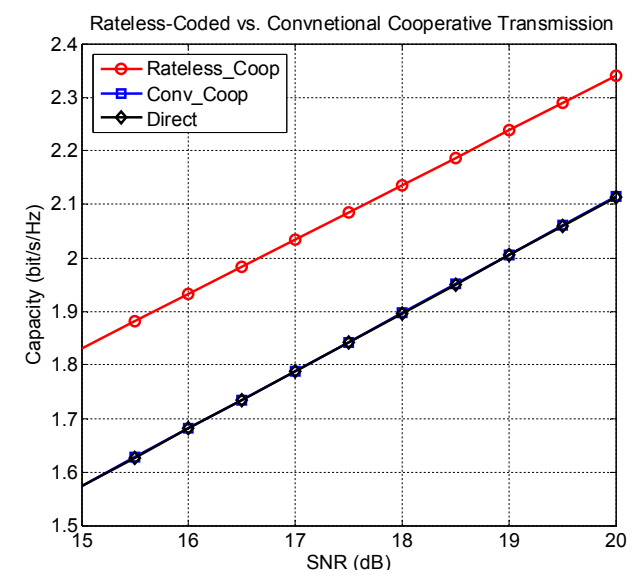

Figure 3. Capacity comparison between rateless-coded and conventional cooperative transmission at high SNR regime.
Figure 4 and Figure 5 show the capacity comparison of the rateless-coded cooperation by different relay selection schemes. In both figures, Proposed Scheme refers to the our proposed relay selection scheme described in Section II and III; Conv. Scheme 1 refers to the relay selection scheme for conventional two-hop transmission [12]; and Conv. Scheme 2 refers to the relay selection scheme for conventional cooperative transmission [11]. We have evaluated the achievable rates of the different relay selection schemes by Monte Carlo simulations. For 10,000 simulations we observed that in over 40 percent cases our proposed selection scheme and those conventional selection schemes result in different relays selected for participating in the cooperative transmission. This is due to different selection criterion employed in each scheme, which is essentially dependent on the way how signals from the source and the relay are processed by the destination. In Figure 4 we observe the following two features. Firstly, at the low SNR regime, the rateless-coded transmission, with any relay selection criterion, outperforms the direct transmission. Secondly, Rateless-coded cooperation by our proposed relay selection scheme constantly outperforms that by both conventional relay selection schemes. This indicates that selecting a relay by conventional schemes results in suboptimal performance for rateless-coded cooperation. The optimal performance is obtained by selecting the best relay by our proposed relay selection scheme. This is attributed to the fact that our proposed relay selection scheme inherently exploits the characteristics of rateless-coded cooperation.

In Figure 5 we observe the following three features. Firstly, at the high SNR regime, the rateless-coded cooperative transmission employing the conventional cooperative relay selection criterion actually reverts to direct transmission, because the conventional relay selection schemes naturally result in none positive relay selected when source-destination channel is strong enough. In other words, the rateless-coded cooperative transmission scheme employing the conventional relay selection criterion in [11] is no longer superior to the direct transmission scheme. Secondly, the rateless-coded cooperative transmission employing the conventional two-hop relay selection scheme underperforms the direct transmission, since in this case no mutual information is accumulated from the direct source-destination link. In effect, the destination starts accumulating mutual information after the selected relay successfully decodes, and the destination only accumulates mutual information from the relay-destination link. Finally, our proposed relay selection scheme results in the best performance among all transmissions, which further justify our proposal.

\section{CONCLUSION}

In this paper, we have devised a relay selection procedure and derived the associated relay selection criterion applicable to rateless-coded cooperative relaying. By the proposed relay selection scheme, a relay capable of giving rise to the highest transmission efficiency is opportunistically selected to participate in the cooperative transmission. Simulation results demonstrate that the system capacity can be enhanced by our scheme compared to using conventional relay selection schemes, and thus justifies our scheme. 


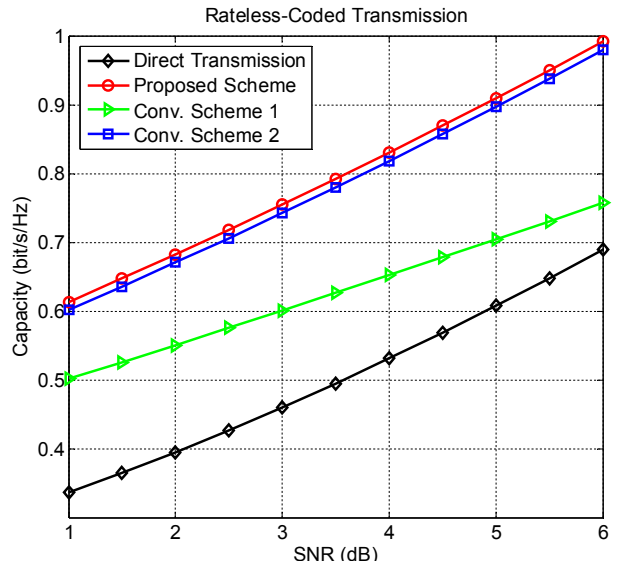

Figure 4. Capacity comparison of rateless-coded transmission with different relay selection schemes at low SNR regime.

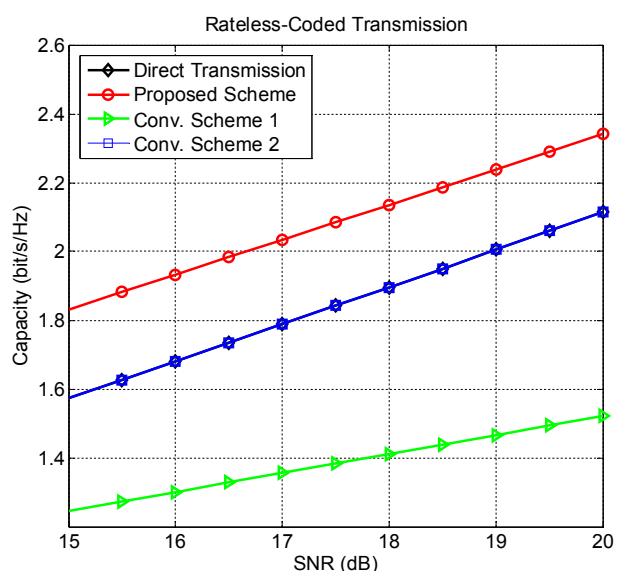

Figure 5. Capacity comparison of rateless-coded transmission with different relay selection schemes at high SNR regime.

\section{ACKNOWLEDGEMENT}

This work was supported in part by the National High Technology Development 863 Program of China under Grant 2009AA011801.

\section{REFERENCES}

[1] J. N. Laneman, D. N. C. Tse, and G. W. Wornell, "Cooperative Diversity in Wireless Neworks: Efficient Protocols and Outage Behavior," IEEE Trans. Inform. Theory, vol. 50, no. 12, pp. 3062-3080, Dec. 2004.

[2] A. Chakrabarti, A. Sabharwal, and B. Aazhang, "Cooperative communications: Fundamental limits and practical implementation," in Cooperation in Wireless Networks: Principles and Applications, F. H. P. Fitzek and M. D. Katz, Eds. Springer, 2006.

[3] P. Liu, Z. Tao, Z. Lin, E. Erkip, and S. Panwar, "Cooperative Wireless communications: A Cross-Layer Approach," IEEE Wireless Commun., vol. 13, no. 4, pp. 84-92, Aug. 2006.

[4] D. J. C. Mackay, "Fountain codes," IEE Proc.-Commun., vol. 152, no. 6, pp. 1062-1068, Dec. 2005

[5] J. Castura and Y. Mao, "Rateless coding for wireless relay channels," IEEE Trans. Wireless Commun., vol. 6, no. 5, pp. 1638-1642, May 2007.

[6] A. F. Molisch, N. B. Mehta, J. Yedidia, and J. Zhang, "Performance of fountain codes in collaborative relay networks," IEEE Trans. Wireless Commun., vol. 6, pp. 4108-4119, Nov. 2007.

[7] Y. Liu, "A low complexity protocol for relay channels employing rateless codes and acknowledgement," in Proc. IEEE ISIT 2006, pp. 914, Jul. 2006.

[8] Y. Liu, M. K. Varanasi, and X. Huang, "Power Scheduling for MIMO Relay Channels Employing Rateless Codes," in Proc. IEEE ICC 2008, pp. 4431-4435, May 2008.

[9] R. Nikjah and N. C. Beaulieu, "Novel rateless coded selection cooperation in dual-hop relaying systems," in Proc. IEEE Globecom 2008, pp. 1-6, Nov. 2008.

[10] R. Nikjah and N. C. Beaulieu, "Achievable rates and fairness in rateless coded relaying schemes," IEEE Trans. Wireless Commun., vol. 7, no. 11 pp. 4439-4444, Nov. 2008.

[11] R. Nikjah and N. C. Beaulieu, "Achievable Rates and Fairness in Rateless Coded Decode-and-Forward Half-Duplex and Full-Duplex Opportunistic Relaying," in Proc. IEEE ICC 2008, pp. 3701-3707, May 2008.

[12] A. Bletsas, A. Khisti, D. Reed, and A. Lippman, "A Simple Cooperative Diversity Method based on Network Path Selection," IEEE J. Sel. Areas Commun., vol. 24, pp. 659-672, Mar. 2006.

[13] S. Song, K. Son, H. Lee, and S. Chong, "Opportunistic Relaying in Cellular Network for Capacity and Fairness Improvement," in Proc. IEEE Globecom 2007, pp. 4407-4412, Nov. 2007.

[14] J. Cai, X. Shen, J. W. Mark, and A. S. Alfa, "Semi-Distributed User Relaying Algorithm for Amplify-and-Forward Wireless Relay Networks," IEEE Trans. Wireless Commun., vol. 7, no. 4, pp. 1348-1357, Apr. 2008.

[15] C. K. Lo, W. Heath, and S. Vishwanath, "Opportunistic Relay Selection with Limited Feedback," in Proc. IEEE VTC 2007-Spring, pp. 135-139, Apr. 2007. 